\title{
Binding of His-tagged fluorophores to lipid bilayers and giant vesicles
}

\author{
Shreya Pramanik ${ }^{a}$, Jan Steinkühler ${ }^{a, b}$, Rumiana Dimova ${ }^{a}$, Joachim Spatz $^{c}$, and Reinhard Lipowsky ${ }^{a, *}$
}

ABSTRACT

His-tagged molecules can be attached to lipid bilayers via certain anchor lipids, a method that has been widely used for the biofunctionalization of membranes and vesicles. To measure the coverage by the membrane-bound molecules, it is useful to study molecules that are fluorescent as well. Here, we use two such molecules, green fluorescence protein (GFP) and green-fluorescent fluorescin isothiocyanate (FITC), both of which are tagged with a chain of six histidines that bind to achor lipids within the bilayers. This His-tag is much smaller in size than the GFP molecule but somewhat larger than the FITC dye. The lipid bilayers form giant unilamellar vesicles (GUVs), the behavior of which can be directly observed in the optical microscope. Several protocols for the preparation of GUVs have been developed. We apply and compare three well-established protocols based on polyvinyl alcohol (PVA) hydrogel swelling, electroformation on platinum wires, and electroformation on indium tin oxide (ITO) glass. For the same nanomolar concentration in the exterior solution, the coverage by His-tagged FITC is much lower than the one by His-tagged GFP. However, for both GFP and FITC, we find that the binding of the His-tagged molecules to the anchor lipids depends strongly on the preparation method. The highest binding affinitiy is obtained for electroformation on platinum wires. PVA gel swelling gives rise to a somewhat smaller binding affinity whereas electroformation on ITO glass leads to essentially no binding. Furthermore, the binding affinitiy is also observed to depend on the $\mathrm{pH}$ of the aqueous solution, with a relatively weak and strong pH-dependence for His-tagged GFP and His-tagged FITC, respectively.

\section{Introduction}

Lipid bilayers, which represent a universal building block for all biomembranes and, thus, for the architecture of the living cell, provide a versatile biomimetic module to understand the mechanical properties of cells and organelles as well as their responses to external stimuli. In aqueous solutions, lipid bilayers form closed vesicles which have a wide range of applications in the field of synthetic biology 115 , intracellular cargo transport and trafficking 6 ․ 9 and the systematic study of biomembrane properties ${ }^{10+13}$. From an experimental point of view, giant unilamellar vesicles (GUVs) are particularly useful because they are micrometer-sized, close to the size of eukaryotic cells. In addition, GUVs can be directly imaged by optical microscopy and their mechanical responses can be probed by optical tweezers and micropipettes $\frac{14}{14}$

Two broad categories of membrane proteins can be distinguished. First, integral membrane proteins have one or several transbilayer domains, which span the hydrophobic core of the bilayer membranes. Second, peripheral membrane proteins, which take part in cell signaling and membrane trafficking, can attach to one leaflet of the bilayer membranes by binding to a specific lipid or to a small cluster of several lipids $\frac{15}{11}$. Likewise, to achieve biofunctionalisation of GUVs, integral membrane proteins are inserted into the membranes using detergents $\frac{16}{16}$ or proteoliposomes $\frac{1718}{18}$ whereas peripheral membrane proteins are attached via certain anchor lipids. Here, we will consider a specific lipid anchor as provided by DGS-NTA(Ni) or NTA lipid for short, ${ }^{\circ}$ which binds to poly-histidine tagged molecules via coordinate bonds. Two such molecules will be considered, His-tagged GFP and His-tagged FITC, both of which are green fluorescent, see Figure 1

Poly-histidine tags are generally attached to one of the terminals of the proteins for their purification 19 . Hence, this specific interaction can be harnessed to attach proteins to lipid bilayers $316|20| 24$. The NTA(Ni) in the lipid head group forms an octahedral coordinate complex with poly-histidine chains. Four vertices of the octahedron are occupied by the NTA group, leaving two vertices for the binding of two imidazole side chains, see Figure 2. The nitrogen from the imidazole side chain of histidine donates electrons for the coordinate bond. ${ }^{20}$ The optimal length of the histidine chain to bind to NTA(Ni) lipids consists of six residues. ${ }^{3}$

The binding between His-tagged proteins and NTA anchor lipids has been frequently used in previous studies. Examples include protein crowding on vesicles ${ }^{25}$, lipid-coated substrates for drug delivery systems ${ }^{26}$, artificial cell adhesion 27 , and high spontaneous curvature generated by the dilute concentration of surface proteins ${ }^{28}$. In general, the $\mathrm{Ni}^{2+}$ ion can be replaced by a $\mathrm{Co}^{3+}$ ion in the NTA group. Poly-histidine chains form a stronger and less labile coordinate bond with NTA-Co ${ }^{3+}$ compared to the corresponding bond with NTA-Ni ${ }^{2+} .29-31$

\footnotetext{
${ }^{a}$ Department of Theory and Bio-Systems, Max Planck Institute of Colloids and Interfaces, Science Park Golm, 14476 Potsdam,Germany

${ }^{b}$ Center for Synthetic Biology, Northwestern University, Evanston, USA

${ }^{c}$ Max Planck Institute for Medical Research, 69120 Heidelberg, Germany

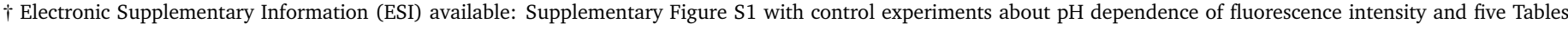

S1 - S5 with numerical values of the data displayed in Figures 5-7] and 9 See DOI: 10.1039/cXsm00000x/

* For chemical formulas, see the list of abbreviations at the end of this article.
} 


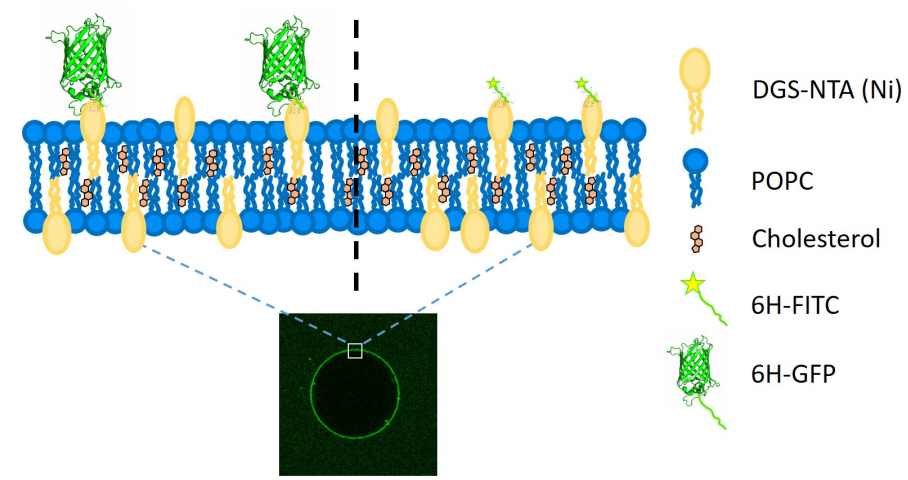

Fig. 1: Cartoon depicting the lipid bilayer composed of POPC, cholesterol, and DGS-NTA(Ni) or NTA lipid, which acts as an anchor for a poly-histidine chain. Two types of molecules tagged with a chain of six histidines will be studied, 6H-GFP and the 6H-FITC, both of which are green fluorescent. The barrel height of 6H-GFP is comparable to the membrane thickness (left) whereas the linear extension of $6 \mathrm{H}$-FITC is governed by the length of the histidine chain (right).

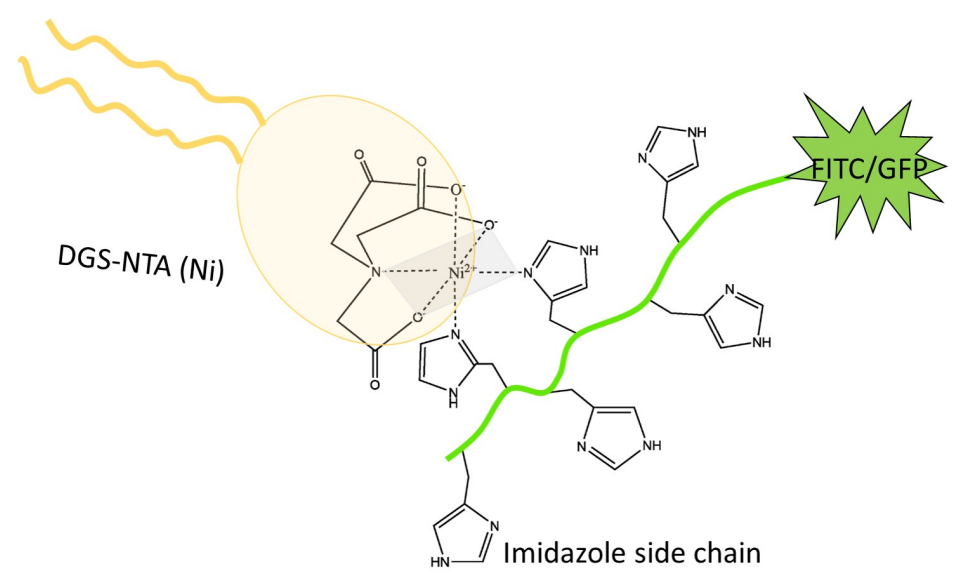

Fig. 2: Both 6H-FITC and 6H-GFP consist of a green fluorescent dye attached to a chain of six histidines with six side chains, corresponding to six imidazole groups. Two of the latter groups interact wth the head group of the DGS-NTA(Ni) lipid forming an octahedral complex. Four vertices of this octahedron (grev) are occupied by three oxygens and one nitrogen from the head group, two vertices by two nitrogens from two imidazole side chains. 19

GUVs can be prepared using a variety of well-established protocols such as swelling lipid layers on a substrate by hydration, electroformation $^{32}$, phase transfer across liquid-liquid interfaces ${ }^{33} \sqrt[34]{ }$, and droplet stabilized compartments ${ }^{22}$; for a recent review, see Ref 14. All of these techniques have their advantages and disadvantages. The swelling methods involve the spreading of a lipid mixture on a substrate followed by the evaporation of the (organic) solvent. As a result, one obtains a stack of lipid bilayers which can then be swollen by hydration. Spontaneous swelling is achieved by spreading the lipid on a non-reactive surface and gently hydrating it overnight ${ }^{35}$. This gentle hydration method is limited to the use of specific lipids and solution conditions ${ }^{36}$.

The influx of the hydrating solution can be enhanced by hydrogel-assisted swelling 36 [37. The lipid film is deposited on the surface of the dried hydrogel. The hydrogels absorbs the hydrating solution and GUVs form rapidly at the hydrogel-aqueous solution interface. The hydrogels typically used for this purpose are agarose ${ }^{37 / 38}$, polyvinyl alcohol (PVA) ${ }^{2836]}$, and polyaralymide gels ${ }^{40}$. Sometimes the hydrogel becomes incorporated into the lumen of the GUV or even within the lipid bilayer, which represents a drawback of this method because it affects the mechanical bilayer properties $38 / 40$. To avoid these unwanted side effects, electroformation can be used to control the hydration of the lipid films by external alternating currents (ACs) 32 . The substrate for spreading the lipid film must be electrically conductive. Commonly used substrates include platinum wires ${ }^{32 / 41}$, ITO coated glasses ${ }^{42} \sqrt[44]{4}$, stainless steel wires ${ }^{45 / 46}$, copper electrodes 47 , and carbon fiber microelectrodes 48 .

One implicit assumption that is often made in the preparation of GUVs is that these vesicles have the same composition as the lipid mixture that was initially used to grow them. This assumption should be checked, especially when the GUVs are prepared from a lipid mixture with a certain fraction of DGS-NTA(Ni). Furthermore, the binding of the poly-histidine with the NTA(Ni) lipid is also sensitive to the solution condition. Here, we will analyze and compare different preparation techniques of GUVs in different pH conditions. As shown in Figures 1 and 2 we will use two different fluorescent probes, FITC and GFP, both of which are tagged by a linear chain of six histidines, and study their binding affinity to NTA lipids embedded in GUV membranes. 
bioRxiv preprint doi: https://doi.org/10.1101/2022.02.01.478643; this version posted February 3, 2022. The copyright holder for this preprint (which was not certified by peer review) is the author/funder, who has granted bioRxiv a license to display the preprint in perpetuity. It is made available under aCC-BY-NC-ND 4.0 International license.

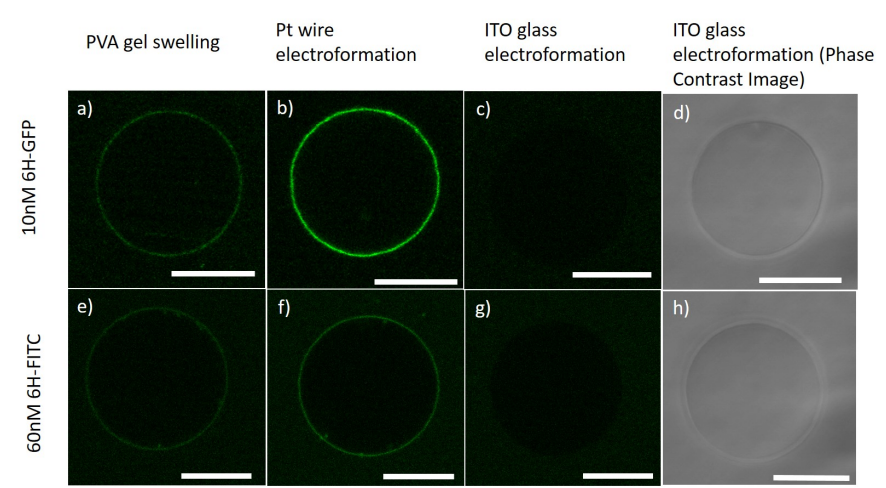

Fig. 3: The binding of His-tagged molecules to GUV membranes doped with DGS-NTA(Ni) lipid depends on the method of preparation. The binding affinity of 6H-GFP to the membrane (a,b) is stronger compared to that of $6 \mathrm{H}$-FITC $(\mathrm{e}, \mathrm{f})$. (c,g)The ITO glass electroformaton leads to GUVs with very weak binding to both types of His-tagged molecules. The lipid composition used for the GUVs is POPC:Chol (8:2) and 3 mol\% DGS-NTA(Ni). In all cases, the GUV encloses a $50 \mathrm{mM}$ sucrose solution and is exposed to an exterior solution of $22.5 \mathrm{mM} \mathrm{NaCl}$ and $5 \mathrm{mM}$ sucrose at pH 7.45 with either $6 \mathrm{H}$-GFP or 6H-FITC. All scale bars: $20 \mu \mathrm{m}$.
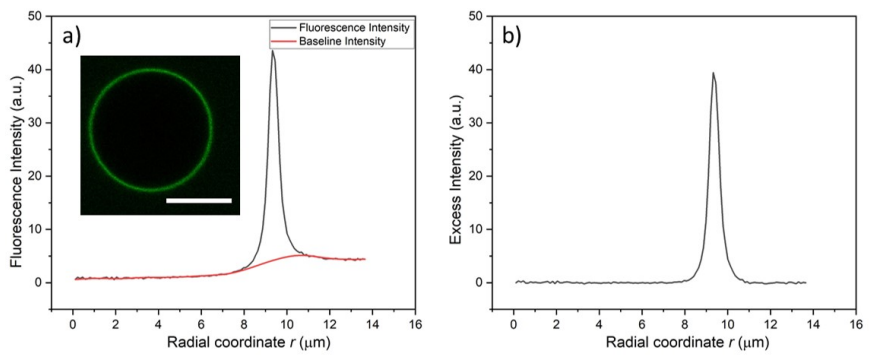

Fig. 4: Measurement of membrane fluorescence from peptides and proteins bound to a GUV membrane: (a) Fuorescence intensity (black) and baseline intensity (red) as a function of the radial coordinate $r$, which measures the distance from the vesicle center. The fluorescence intensity was obtained via the image processing software ImageJ, the baseline intensity via OriginLab; and (b) Excess intensity obtained by substracting the baseline intensity from the fluorescence intensity in (a). The membrane fluorescence is obtained by integrating the excess intensity over the radial coordinate $r$. The intensities were obtained for the vesicle displayed in the inset of (a), corresponding to $3 \mathrm{~mol} \%$ DGS-NTA(Ni), $120 \mathrm{nM} 6 \mathrm{H}-\mathrm{FITC}$, and pH 7.45. Scale bar: $10 \mu \mathrm{m}$.

\section{Results and Discussion}

The DGS-NTA(Ni) or NTA anchor lipid is used for the biofunctionalisation of vesicles. This NTA functionalised lipid head group can selectively attach to poly-histidine end groups of biologically relevant proteins and peptides. The proteins can also be cloned with multiple histidine in the $\mathrm{N}$ or $\mathrm{C}$ terminal so that the binding with the DGS-NTA(Ni) does not interfere with the function of the protein. Because the binding is mediated by a coordinate bond between the head group of the NTA lipid and the imidazole side chains of polyhistidine (Figure 2), one has to pay particular attention to the precise solution conditions such as the $\mathrm{pH}$ and the salt concentrations.

\subsection{Binding of 6H-GFP to GUV membranes}

First, consider the His-tagged molecule 6H-GFP, corresponding to a chain of six histidines attached to the $\mathrm{N}$ terminal of GFP as used in Ref 28. The histidine chain binds to the NTA(Ni) head group of the DGS-NTA(Ni) lipid in the vesicle via a coordinate bond, see Figure 2 Our experiments revealed that the binding of 6H-GFP to the GUV membranes depends strongly on the method used for the preparation of the GUVs. Three different preparation methods were used to form such vesicles: PVA hydrogel swelling, electroformation on platinum wires, and electroformation on ITO glass. In all cases, we started from the same initial lipid mixture with 3 mol\% of DGS-NTA(Ni) to grow the GUVs, which were subsequently exposed to a $20 \mathrm{nM}$ solution of 6H-GFP in a Protein LoBind Eppendorf tube. After 10 mins, the GUVs were directly observed using a confocal microscope, see the snapshots in Figure 3 which were obtained for a $10 \mathrm{nM}$ solution of $6 \mathrm{H}-\mathrm{GFP}$.

Because the initial lipid mixture used for all the GUV-forming methods was the same, the GUVs obtained from the different techniques were expected to have the same properties. Based on this expectation, all vesicles doped with $3 \mathrm{~mol} \%$ of DGS-NTA(Ni) should exhibit a similar binding affinity to 6H-GFP and, thus, a similar brightness when exposed to the same 6H-GFP concentration. However, this brightness was observed to be very different for the different protocols. Indeed, as shown in Figure 3, the GUV membranes formed by electroformation on platinum wire were rather bright, whereas those formed by PVA hydrogel swelling and on ITO glasess exhibited a strongly reduced and almost no fluorescence, respectively.

For a spherical GUV, the membrane fluorescence can be determined quantitatively by measuring the fluorescence intensity profile as a function of the radial coordinate $r$, which represents the distance from the center of the vesicle. One example is shown in Figure $4 \mathrm{~B}$ together with the baseline intensity that interpolates smoothly between the fluorescence intensities of the interior and exterior solutions. 
The excess intensity of the fluorescence is equal to the difference between the measured fluorescence and baseline intensities, see Figure 4 , and the membrane fluorescence is obtained by integrating this excess intensity over the radial coordinate $r$.

Using the calibration method described in the Material and Method section, which is based on Fluorescein-DHPE, we transformed the measured fluorescence of the membrane into the surface coverage of the membrane by 6H-GFP. By definition, the surface coverage is equal to the surface density of membrane-bound 6H-GFP and is given in units of number of membrane-bound molecules per $\mu \mathrm{m}^{2}$. The resulting data for the surface coverage are displayed in Figure 5 and the corresponding mean values and standard deviations are provided in Table S1.

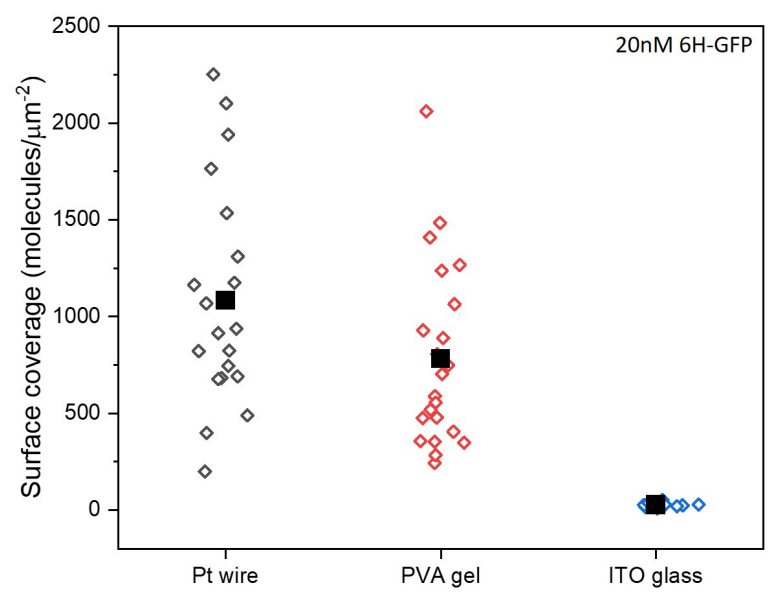

Fig. 5: Coverage of GUV membranes by $6 \mathrm{H}-\mathrm{GFP}$ for different preparation protocols. All GUVs were prepared with 3 mol\% of NTA lipids and were exposed to a $20 \mathrm{nM}$ solution of $6 \mathrm{H}-\mathrm{GFP}$ at $\mathrm{pH} 7.45$. The three sets of data were obtained for 20, 23, and 10 vesicles using the protocol based on Pt wire, PVA gel, and ITO glass, respectively. The solid squares represent the mean values of the coverage obtained for each data set. The numerical values of the mean coverage and the standard deviation are given in Table S1.

The data in Figure 5 and Table S1 confirm our previous conclusion from Figure 3 that GUV membranes prepared by electroformation on platinum wires bind the largest amount of 6H-GFP whereas membranes prepared by PVA hydrogel swelling exhibit a weaker binding affinity for this His-tagged protein and electroformation on ITO glass leads to essentially zero coverge of 6H-GFP. In addition, the data in Figure 5 and Table S1 also show that the preparation based on PVA hydrogel swelling leads to a smaller spread of the surface coverage for different GUVs from the same batch.

Using the three preparation methods, we observe different values of the surface coverage for different vesicles from the same batch. Possible causes for these deviations are that the vesicle membranes contain different mole fractions of NTA lipids and/or that the fraction of NTA lipids with a bound $\mathrm{Ni}^{2+}$ ion differs from vesicle to vesicle.

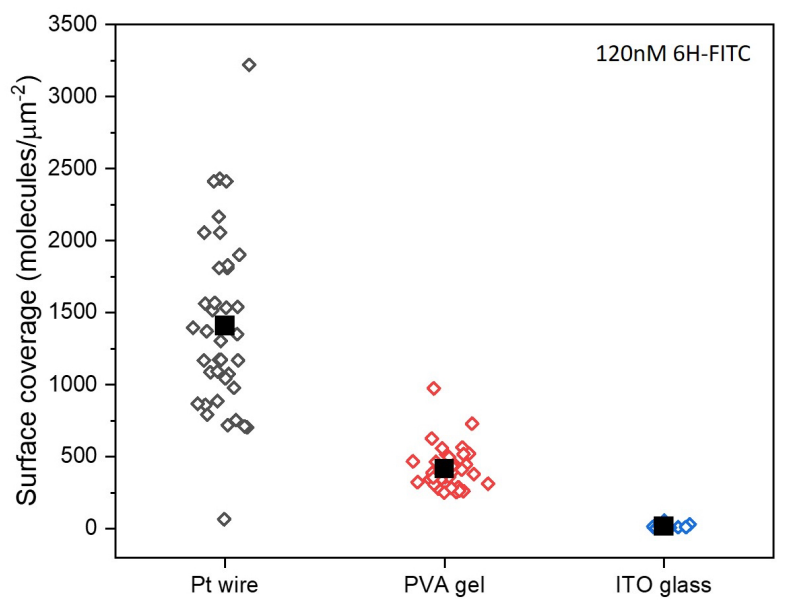

Fig. 6: Coverage of GUV membranes by 6H-FITC for different preparation protocols. All GUVs were prepared with 3 mol\% of NTA lipids and were exposed to a $120 \mathrm{nM}$ solution of $6 \mathrm{H}$-FITC at $\mathrm{pH} 7.45$. The three sets of data were obtained for 38 , 38, and 10 vesicles using the protocol based on Pt wire, PVA gel, and ITO glass, respectively. The solid squares represent the mean values of the coverage for each data set. The numerical values of the mean coverage and the standard deviation are provided in Table S2. 


\subsection{Binding of 6H-FITC to GUV membranes}

Next, we studied the His-tagged molecule 6H-FITC, consisting of six histidines attached to the small fluorescent probe FITC. We used the same experimental protocol to determine membrane fluorescence and surface coverage as described in the previous section for $6 \mathrm{H}$-GFP. Because we observed a reduced binding affinity of $6 \mathrm{H}$-FITC compared to $6 \mathrm{H}$-GFP, see the images in Figure 3 , we exposed the GUVs to $120 \mathrm{nM}$ solutions of 6H-FITC, corresponding to a six-fold increase in the solution concentration compared to 6H-GFP.

Inspection of Figure6 6 shows that the preparation method based on the Pt wire protocol leads to the largest mean value of the coverage by 6H-FITC. The mean coverage obtained via the PVA gel method is reduced to about one third of the mean coverage from the Pt wire method. On the other hand, the data from the PVA method exhibit a relatively small spread compared to those from the Pt wire protocol. Finally, the protocal based on ITO glass leads to essentially zero coverage by 6 H-FITC. The numerical values of the mean coverage and of the standard deviatiom for the coverage are given in Table S2.

Comparison of Figure 6 with Figure 5 reveals that the three preparation protocols lead to similar trends for both His-tagged GFP and His-tagged FITC. In both cases, the coverage obtained from the ITO glass preparation is very low, the largest mean coverage is observed for the Pt wire protocol and the smallest spread of the data from the PVA gel method.

The reduced binding affinity of 6H-FITC can be understood as follows. The two coordinate bonds formed between the NTA lipid and the His-tag take place sequentially 20 . One of the imidazole side chains, see Figure2 makes contact with the head group of an NTA lipid, which brings a second imidazole side chain closer to the same head group. 6H-FITC is a relatively small molecule that undergoes fast thermal motion, which can lead to a break-up of the first coordinate bond before the second bond has been formed. Because 6H-GFP is much larger, its translational and rotational diffusion is reduced which facilitates the formation of the second bond. Different molecular interactions between the bilayer and His-tagged molecules might also play a role in the reduced affinity of 6H-FITC compared to $6 \mathrm{H}$-GFP as proposed for other types of His-tagged proteins 49 .

\subsection{Dependence on mole fraction of NTA lipids}

From the comparison of the three vesicle preparation protocols, we conclude that the His-tagged molecules exhibit the smallest binding affinity to the GUV membranes when the GUVs were prepared by electroformation on ITO glass. The hydrogel assisted swelling of vesicles with the help of PVA produced GUVs in a very short time. However, when harvesting these GUVs, we typically obtained 'debris' of PVA in the final GUV solution. When this sample is exposed to His-tagged molecules, the PVA debris tends to bind these molecules as well, thereby decreasing the bulk concentration of the molecules. PVA can also become incorporated into the lipid bilayer which changes the bilayer properties $50 \mid 51$.

To get additional insight into the weak binding affinity of His-tagged molecules to GUVs that were grown by electroformation on ITO glasses, we compared this method with electroformation on platinum wires, using two different lipid mixtures with an enlarged mole fraction of $10 \mathrm{~mol} \%$ and $30 \mathrm{~mol} \%$ DGS-NTA(Ni) lipids. After harvesting the GUVs, they were exposed to $20 \mathrm{nM} 6 \mathrm{H}-\mathrm{GFP}$ in the exterior solution. As shown in Figure7 7 we observe that electroformation of GUVs on ITO glass exhibits weak binding to 6H-GFP. However, the corresponding binding affinity is low compared to the vesicles grown by platinum wire electroformation with the same lipid composition. This indicates that only a fraction of the NTA lipid takes part in the vesicle formation and that the remaining NTA is "lost".
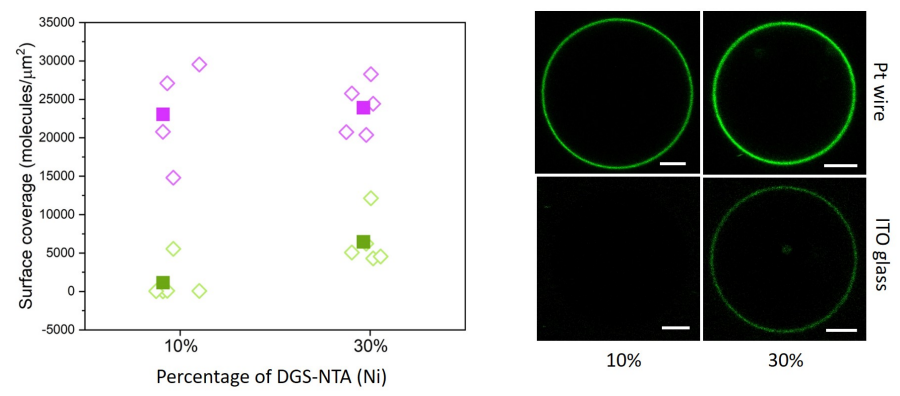

Fig. 7: Binding of 6H-GFP to GUV membranes with $10 \mathrm{~mol} \%$ and $30 \mathrm{~mol} \%$ DGS-NTA(Ni) anchor lipid: (a) The GUVs were formed using Pt wire electroformation (purple) and ITO glass electroformation (green), and subsequently exposed to a $20 \mathrm{nM}$ solution of $6 \mathrm{H}$-GFP. The filled squares represent the mean values of the respective data. The numerical values of these data are given in Table S3; and (b) Representative images of GUVs taken for the different preparation protocols. The fluorescence intensities of the GUV membranes grown on ITO glasses is low compared to those grown on platinum wire, indicating that only a fraction of the DGS-NTA(Ni) lipid deposited on the ITO glass takes becomes incorporated into the vesicle. All scale bars are $5 \mu \mathrm{m}$

After the deposition of lipid stock on the ITO glass surface, the lipid molecules have orientational freedom to arrange themselves into bilayers 42 . The surface properties of the ITO glass are likely to affect the properties of the produced vesicles ${ }^{52}$. The ITO film consists of Indium(III) ions, which can bind to NTA 53 55. Presumably, the NTA lipid prefers to stay close to the ITO surface and forms a weak bond with indium, when the lipid stock dissolved in chloroform is deposited on the ITO surface, When the fraction of NTA is increased in the lipid stock to $30 \mathrm{~mol} \%$, the ITO surface becomes saturated and some excess NTA is then incorporated into the vesicle 
bioRxiv preprint doi: https://doi.org/10.1101/2022.02.01.478643; this version posted February 3, 2022. The copyright holder for this preprint (which was not certified by peer review) is the author/funder, who has granted bioRxiv a license to display the preprint in perpetuity. It is made available under aCC-BY-NC-ND 4.0 International license.

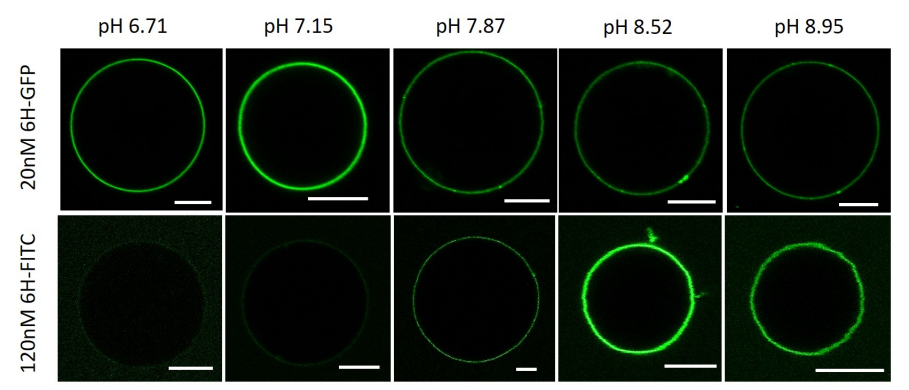

Fig. 8: Dependence of the binding affinity between GUV membranes with $3 \mathrm{~mol} \%$ NTA lipids and two types of His-tagged probes, $6 \mathrm{H}-\mathrm{GFP}$ and $6 \mathrm{H}-\mathrm{FITC}$, on the $\mathrm{pH}$ of the exterior solution, as visualized by the brightness of the membrane fluorescence: GUVs exposed to $20 \mathrm{nM}$ solution of $6 \mathrm{H}$-GFP (top row) and $120 \mathrm{nM}$ of $6 \mathrm{H}$-FITC (bottom row). All scale bars: $10 \mu \mathrm{m}$.
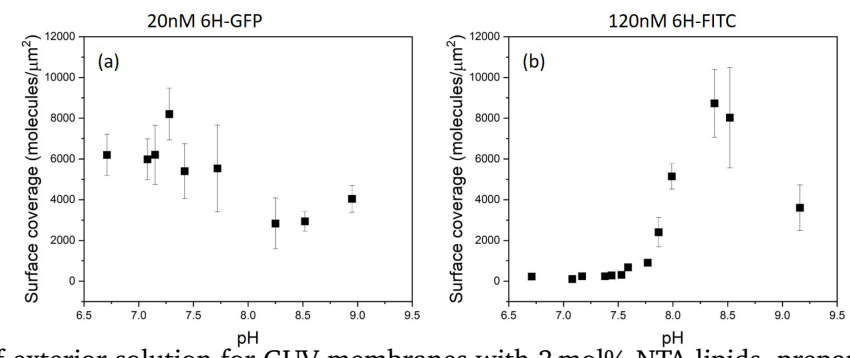

Fig. 9: Surface coverage versus $\mathrm{pH}$ of exterior solution for GUV membranes with $3 \mathrm{~mol} \%$ NTA lipids, prepared by electroformation on platinum wires: (a) For GUVs exposed to $20 \mathrm{nM} 6 \mathrm{H}-\mathrm{GFP}$, the surface coverage exhibits a weak pH dependence with a maximum around pH 7.28; and (b) For GUVs exposed to $120 \mathrm{nM} 6 \mathrm{H}$-FITC, the surface coverage exhibits a strong $\mathrm{pH}$ dependence with a pronounced maximum around $\mathrm{pH} 8.38$. The numerical values of the data are given in Tables S4 and S5. Control experiments show that the pH dependence in (a) and (b) arises from the pH dependence of the His-NTA binding affinity, see Figure S1.

membrane.

\subsection{Dependence of binding affinity on $\mathrm{pH}$}

Our experiments also showed that the binding affinity of His-tagged molecules to NTA anchor lipids can depend strongly on the $\mathrm{pH}$ value of the exterior aqueous solution. This dependence is visualized in Figure 8 via the brightness of the GUV membranes. Inspection of this figure reveals that the pH-dependence of the binding affinity is quite different for 6H-GFP and 6H-FITC.

To determine the $\mathrm{pH}$-dependence of the binding affinity in a systematic manner, the vesicles were grown using platinum wire electrofromation. The swelling solution consisted of $50 \mathrm{mM}$ sucrose and $2 \mathrm{mM}$ HEPES buffer at $\mathrm{pH}$ 7.4. The vesicles were then exposed to an exterior solution containing $20 \mathrm{nM} 6 \mathrm{H}$-GFP or $120 \mathrm{nM} 6 \mathrm{H}$-FITC as well as $22.5 \mathrm{mM} \mathrm{NaCl}, 5 \mathrm{mM}$ sucrose and $2 \mathrm{mM}$ HEPES buffer. The $\mathrm{pH}$ of these solutions was varied by adding small concentrations of $\mathrm{NaOH}$ solutions. For each $\mathrm{pH}$ value, the membrane fluorescence was determined via the method described in Figure 4 and the surface coverage was then obtained using the calibration via FluoresceinDHPE.

The corresponding coverage of the GUV membranes by the two types of His-tagged molecules is shown in Figure9p and 9p. For 6H-GFP, we observe a relatively weak dependence of the coverage on the $\mathrm{pH}$ value with a maximum at about $\mathrm{pH} 7.28$. In contrast, for $6 \mathrm{H}$-FITC, the coverage depends strongly on the $\mathrm{pH}$ value with a pronounced maximum at about $\mathrm{pH} 8.38$, see the data in Tables $\mathrm{S} 4$ and S5. .

In general, the fluorescence of a dye molecule can vary with the $\mathrm{pH}$ value even in the absence of lipids. In order to determine the latter $\mathrm{pH}$ dependence, we also measured the background fluorescence intensities for 6H-GFP and 6H-FITC in the absence of vesicles. The corresponding data are displayed in Figure S1. Inspection of Figure S1b shows that the background intensities obtained for 6H-FITC vary by less than a factor of three when the $\mathrm{pH}$ is varied from 6.5 to 9.0, which is in strong contrast to the surface coverage data shown in Figure $9 \mathrm{p}$. Furthermore, when the membrane-bound dye molecules produced a significant peak intensity, this intensity was always much larger than the background intensities. Therefore, the $\mathrm{pH}$ dependence for 6H-FITC and 6H-GFP as displayed in Figures 8 and 9 reflects the $\mathrm{pH}$ dependence of the His-NTA binding affinity rather than the intrinsic $\mathrm{pH}$ dependence of the dye molecules in solution.

\section{Conclusions}

The binding of His-tagged molecules to GUV membranes has been investigated, using the anchor lipids DGS-NTA(Ni) that form coordinate bonds with the His-tags. Two different green fluorescent molecules, GFP and FITC, have been studied, both of which were tagged to a chain of six histidines. The GUVs were prepared using three well-established protocols provided by PVA hydrogel swelling, electroformation on platinum wires, and electroformation on ITO glass, see the corresponding panels in Figure 3 For all three protocols, 
the lipid mixture used to prepare the GUVs contained $3 \mathrm{~mol} \%$ of the NTA anchor lipid. In all three cases, the binding affinity of 6H-FITC was observed to be small compared to the affinity of 6H-GFP as follows directly from the brightness of the GUV membranes in Figure 3 . taking into account that the GUVs were exposed to a 6H-FITC concentration that was six times as large as the 6H-GFP concentration. However, our experiments also demonstrated that the binding of the His-tagged molecules strongly depends on the preparation method.

To determine the coverage of the membranes by the His-tagged molecules in a quantitative manner, we measured the fluorescence intensity profiles, see Figure 4 , for several batches of GUVs. The resulting data for the surface coverage by 6H-GFP and 6H-FITC are displayed in Figures 5 and 6 , respectively. Comparing the results for the three preparation protocols, we conclude that the strongest binding affinity was obtained when the GUVs were prepared by platinum wire electroformation, a somewhat smaller binding affinity from the PVA gel swelling method, and essentially no binding for the electroformation on ITO glass. The latter results apply to $3 \mathrm{~mol} \%$ NTA anchor lipid. However, when we increase the mole fraction of the anchor lipids from $3 \mathrm{~mol} \%$ to $30 \mathrm{~mol} \%$, we start to detect a layer of His-tagged molecules bound to the GUV membranes, see Figure7 These observations indicate that the ITO glass surface has a certain storage capacity for the His-tagged molecules and that these molecules start to become incorporated into the lipid bilayers after the ITO surface has been saturated.

Another surprising outcome of our study is that it reveals a rather different $\mathrm{pH}$ dependence of the binding affinities for 6H-GFP and 6H-FITC, see Figures 8 and 9 For 6H-GFP, we observe a relatively weak dependence of the surface coverage on the $\mathrm{pH}$ value with a maximum at about pH 7.3 (Figure9 9 ). For 6H-FITC, on the other hand, the coverage depends strongly on the pH value with a pronounced maximum at about $\mathrm{pH} 8.5$ (Figure 9 ). Furthermore, when the membrane-bound dye molecules produced a significant fluorescence intensity, this peak intensity was always much larger than the background intensities, corresponding to those dye molecules in the exterior solution that were not in contact with the lipid membranes, see the example in Figure $4 \mathrm{k}$. In addition, the background intensities for 6H-FITC vary by less than a factor of three over the whole range of $\mathrm{pH}$ values that has been studied (Figure S1b), whereas the surface coverage by $6 \mathrm{H}-\mathrm{FiTC}$ varies by two orders of magnitude (Figure $9 \mathrm{p}$ ). Therefore, the $\mathrm{pH}$ dependence displayed in Figures 8 and 9 reflects the $\mathrm{pH}$ dependence of the His-NTA binding affinity rather than the intrinsic $\mathrm{pH}$ dependence of the fluorescent dye molecules in solution.

Previous experiments have shown that GUV membranes exposed to nanomolar solutions of 6H-GFP aquire a large spontaneous curvature that can be fine-tuned to divide the GUVs in a controlled manner. $\frac{28}{2}$ In the latter experiments, a buffer with $\mathrm{pH} 7.3$ has been used. For this $\mathrm{pH}$ value, the coverage by $6 \mathrm{H}$-FITC is much smaller than the one by $6 \mathrm{H}$-GFP, as follows from the comparison of the data in Figure $9 \mathrm{~g}$ with those in Figure $9 \mathrm{p}$. Therefore, we conclude that, for $\mathrm{pH} 7.3$, it will be rather difficult to generate a sufficiently large spontaneous curvature by the binding of $6 \mathrm{H}$-FITC to achieve a controlled division of the GUVs. On the other hand, the surface coverage by 6H-FITC can be increased by several orders of magnitude, when we consider an exterior buffer with pH 8.4, see Figure 9 p. Therefore, it will be interesting to study the spontaneous curvature generated by membrane-bound 6H-FITC for increased $\mathrm{pH}$ values around $\mathrm{pH} 8.4$.

\section{Material and Method}

\subsection{Giant Unilamellar Vesicles}

Chloroform stock solutions of 1-palmitoyl-2-oleoyl-sn-glycero-3-phosphocholine (POPC) and cholesterol were mixed at a molar ratio of 8:2 with a final lipid concentration of $4 \mathrm{mM}$. The required mol\% of DGS-NTA (Ni) was added to the lipid stock solution. This stock was used as it is or diluted depending on the preparation method. All lipids were obtained from Avanti Polar Lipids.

PVA hydrogel swelling A PVA solution was prepared by dissolving $40 \mathrm{mg}$ of PVA in $1 \mathrm{~mL}$ of water. $40 \mu \mathrm{L}$ of this solution was spread on clean cover glasses and dried in an oven at $40^{\circ} \mathrm{C}$ for $30 \mathrm{~min} .4 \mu \mathrm{L}$ of $2 \mathrm{mM}$ lipid mixture consisting of POPC, cholesterol, and NTA was spread and kept under vacuum for one hour to eliminate trace amounts of chloroform. A closed chamber was created using a Teflon spacer and another cover glass. An aqueous solution of $50 \mathrm{mM}$ sucrose in $2 \mathrm{mM}$ HEPES buffer (pH 7.4) was introduced into the chamber to hydrate the lipid film. The vesicles were harvested after gentle tapping on the PVA coated glass.

Electroformation on ITO glasses. $4 \mu \mathrm{L}$ of a $4 \mathrm{mM}$ solution of the same lipid mixture was spread on two ITO coated glasses using a syringe. The resulting lipid films formed bilayers stacks on the ITO glass surfaces, which were then kept under vacuum for an hour to remove remaining traces of chloroform. The two glasses were then arranged into a closed chamber by using a Teflon spacer as side walls. The chamber was filled with $50 \mathrm{mM}$ of sucrose in $2 \mathrm{mM}$ HEPES buffer (pH 7.4). An AC electric field with peak-to-peak voltage of $2 \mathrm{~V}$ and frequency of $10 \mathrm{~Hz}$ was applied for 2 hours at room temperature. Subsequently, the frequency of the AC field was reduced to $3 \mathrm{~Hz}$ and the low-frequency field was applied for $5 \mathrm{~min}$, to detach the vesicles from the glass surfaces. The vesicles were harvested using a micropipette with a broad tip.

Electroformation on platinum wires. $6 \mu \mathrm{L}$ of a $0.5 \mathrm{mM}$ solution of the same lipid mixture was spread on two platinum wires using a syringe. The wires were kept under vacuum for an hour to remove traces of chloroform. The wires were then dipped in a quartz cuvette, which was filled with $50 \mathrm{mM}$ of sucrose in $2 \mathrm{mM}$ HEPES buffer (pH 7.4). An AC electric field with peak-to-peak voltage of $3 \mathrm{~V}$ and frequency of $10 \mathrm{~Hz}$ was applied for 2 hours at $35^{\circ} \mathrm{C}$. Subsequently, the frequency of the AC field was reduced to $3 \mathrm{~Hz}$ and the low-frequency field was applied for $5 \mathrm{~min}$, to detach the vesicles from the wires. After cooling to room temperature, the vesicles were 
harvested using a micropipette with a broad tip.

\subsection{Image acquisition}

All fluorescent images were recorded using a Leica SP5 and a Leica SP8 confocal microscope. Argon laser with wavelength $488 \mathrm{~nm}$ was used to excite both the GFP proteins and the FITC dye. The emission was collected in the range of wavelengths from 495 to $550 \mathrm{~nm}$. The images for quantification of the fluorescence intensity on the membrane were obtained using a 40X 1.3 oil immersion objective.

\subsection{Membrane fluorescence and surface coverage}

Radial Profile Angle from ImageJ was used to plot the fluorescence intensity as a function of the radial coordinate $r$, see Figure 4 a. The peak analyzer of Origin2021b from OriginLab was used to determine the excess intensity in Figure $4 \mathrm{~b}$ by subtracting the baseline intensity from the fluorescence intensity. Then the membrane fliuorescence was obtained by integrating the excess intensity over the radial coordinate $r$, using the whole range of $r$-values.

To transform the membrane fluorescence into the surface coverage of the membranes by 6H-GFP and 6H-FITC, a vesicle doped with $0.1 \mathrm{~mol} \%$ of a fluorescently labeled lipid (Fluorescein-DHPE) was imaged with the same image settings. Because the fluorescein dye is also responsible for the fluorescence of FITC, the Fluorescein-DHPE lipid leads to the same fluorescence intensity as 6H-FITC. For 6H-GFP, the detector gain was adjusted to give the same intensity for the same bulk concentrations of 6H-FITC and 6H-GFP. The image of the vesicle with Fluorescein-DHPE is obtained with the adjusted detector gain.

For the vesicle membranes with $0.1 \mathrm{~mol} \%$ of this lipid, the surface coverage of one bilayer leaflet by Fluorescein-DHPE lipids is calculated and used to calibrate the membrane fluorescence. For the vesicles with peripheral 6H-FITC and 6H-GFP, the surface density is calculated by comparing its intensity with that of the Fluorescein-DHPE containing vesicle. For measuring the surface coverage at different $\mathrm{pH}$ values, the vesicles were grown using platinum wire electroformation at $\mathrm{pH}$ 7.4. These vesicles were then suspended in different aqueous solutions, distinguished by their $\mathrm{pH}$ that was varied from 6.5 to 9.5 by the addition of $\mathrm{NaOH}$ solution. The final $\mathrm{pH}$ that is recorded was measured using a micro $\mathrm{pH}$ electrode from Mettler Toledo.

\section{Abbreviations}

AC: alternating current

DGS-NTA(Ni) or NTA for short: 1,2-dioleoyl-sn-glycero-3-[(N-(5-amino-1-carbox-ypentyl)iminodiacetic acid)succinyl] (nickel salt) Fluorescein-DHPE: N-(Fluorescein-5-thiocarbonyl)-1,2-dihexadecyl-sn-gycero-3-phosphoethanolamine, triethylammonium salt

FITC: Fluorescein isothiocyanate

GFP: green fluoerescent protein

ITO: indium tin oxide

POPC: 1-Palmitoyl-2-oleoyl-sn-glycero-3-phosphocholine

PVA: polyvinyl alcohol

6H-GFP: green fluorescent protein tagged by six histidines

6H-FITC: Fluorescein isothiocyanate tagged by six histidines

\section{Conflicts of interest}

There are no conflicts to declare.

\section{Acknowledgements}

We acknowledge support by the Max Planck School 'Matter to Life'.

\section{Notes and references}

1 C. Schmitt, A. H. Lippert, N. Bonakdar, V. Sandoghdar and L. M. Voll, Frontiers in bioengineering and biotechnology, 2016, 4, 19.

2 K. Kamiya, Micromachines, 2020, 11, 559.

3 L. Van de Cauter, F. Fanalista, L. van Buren, N. De Franceschi, E. Godino, S. Bouw, C. Danelon, C. Dekker, G. H. Koenderink and K. A. Ganzinger, ACS Synthetic Biology, 2021, 10, 1610-1702.

4 J. W. Hindley, R. V. Law and O. Ces, SN Applied Sciences, 2020, 2, 1-10.

5 R. Lipowsky, Advanced Biology, 2022, in press, DOI: 10.1002/adbi.202101020.

6 O. Staufer, S. Antona, D. Zhang, J. Csatári, M. Schröter, J.-W. Janiesch, S. Fabritz, I. Berger, I. Platzman and J. P. Spatz, Biomaterials, 2021, 264, 120203.

7 L. van der Koog, T. B. Gandek and A. Nagelkerke, Advanced healthcare materials, 2021, 2100639. 
8 Y. Lee and D. Thompson, Wiley Interdisciplinary Reviews: Nanomedicine and Nanobiotechnology, 2017, 9, e1450.

9 J. Lou and M. D. Best, Chemistry and Physics of Lipids, 2020, 232, 104966.

10 M. Karimi, J. Steinkühler, D. Roy, R. Dasgupta, R. Lipowsky and R. Dimova, Nano Letters, 2018, 18, 7816-7821.

11 R. Dimova, Annual review of biophysics, 2019, 48, 93-119.

12 S. Aden, T. Snoj and G. Anderluh, Methods in Enzymology, 2021, 649, 219-251.

13 K. Karamdad, R. Law, J. Seddon, N. Brooks and O. Ces, Lab on a Chip, 2015, 15, 557-562.

14 R. Dimova, P. Stano, C. M. Marques and P. Walde, The Giant Vesicle Book, CRC Press, Taylor \& Francis, 2020, pp. 3-20.

15 R. V. Stahelin, Lipid binding domains: More than simple lipid effectors, 2009.

16 M. Dezi, A. Di Cicco, P. Bassereau and D. Lévy, Proceedings of the National Academy of Sciences, 2013, 110, 7276-7281.

17 I. L. Jorgensen, G. C. Kemmer and T. G. Pomorski, Eur. Biophys. J., 2017, 46, 103-119.

18 M. Garten, D. Lévy and P. Bassereau, The Giant Vesicle Book, Taylor \& Francis, 2020, ch. 3.

19 J. A. Bornhorst and J. J. Falke, Methods in enzymology, 2000, 326, 245-254.

20 S. Knecht, D. Ricklin, A. N. Eberle and B. Ernst, Journal of Molecular Recognition, 2009, 270-279.

21 J. A. Nye and J. T. Groves, Langmuir, 2008, 24, 4145-4149.

22 M. Weiss, J. P. Frohnmayer, L. T. Benk, B. Haller, J. W. Janiesch, T. Heitkamp, M. Börsch, R. B. Lira, R. Dimova, R. Lipowsky, E. Bodenschatz, J. C. Baret, T. Vidakovic-Koch, K. Sundmacher, I. Platzman and J. P. Spatz, Nature Materials, 2018, 89-96.

23 S. Deshpande, Y. Caspi, A. E. Meijering and C. Dekker, Nature Communications, 2016, 1-9.

24 K. Dürre and A. R. Bausch, Soft Matter, 2019, 9676-9681.

25 J. C. Stachowiak, E. M. Schmid, C. J. Ryan, H. S. Ann, D. Y. Sasaki, M. B. Sherman, P. L. Geissler, D. A. Fletcher and C. C. Hayden, Nature Cell Biology, 2012, 14, 944-949.

26 M. Farell, A. Self, C. Guza, H. Song, L. Apollon, E. W. Gomez and M. Kumar, ACS Applied Materials and Interfaces, 2020, 1240712416.

27 S. Togo, K. Sato, R. Kawamura, N. Kobayashi, M. Noiri, S. Nakabayashi, Y. Teramura and H. Y. Yoshikawa, APL Bioengineering, 2020, 016103.

28 J. Steinkühler, R. L. Knorr, Z. Zhao, T. Bhatia, S. M. Bartelt, S. Wegner, R. Dimova and R. Lipowsky, Nature Communications, 2020, 11, 905.

29 S. V. Wegner, F. C. Schenk and J. P. Spatz, Chemistry - A European Journal, 2016, 22, 3156-3162.

30 S. V. Wegner and J. P. Spatz, Angewandte Chemie International Edition, 2013, 52, 7593-7596.

31 J. Di Russo, J. L. Young, A. Balakrishnan, A. S. Benk and J. P. Spatz, Biomaterials, 2019, 192, 171-178.

32 M. I. Angelova and D. S. Dimitrov, Faraday Discussions of the Chemical Society, 1986, 303-311.

33 M. Abkarian, E. Loiseau and G. Massiera, Soft Matter, 2011, 7, 4610 - 4614.

34 M. C. Blosser, B. G. Horst and S. L. Keller, Soft Matter, 2016, 12, 7364-7371.

35 J. P. Reeves and R. M. Dowben, Journal of Cellular Physiology, 1969, 49-60.

36 A. Weinberger, F. C. Tsai, G. H. Koenderink, T. F. Schmidt, R. Itri, W. Meier, T. Schmatko, A. Schröder and C. Marques, Biophysical Journal, 2013, 154-164.

37 K. S. Horger, D. J. Estes, R. Capone and M. Mayer, Journal of the American Chemical Society, 2009, 1810-1819.

38 R. B. Lira, R. Dimova and K. A. Riske, Biophysical journal, 2014, 107, 1609-1619. 
39 A. Witkowska, L. Jablonski and R. Jahn, Scientific reports, 2018, 8, 9422.

40 E. Parigoris, D. L. Dunkelmann, A. Murphy, N. Wili, A. Kaech, C. Dumrese, N. Jimenez-Rojo and U. Silvan, Scientific reports, 2020, 10, 1-10.

41 E. M. Schmid, D. L. Richmond and D. A. Fletcher, Methods in Cell Biology, 2015, 319-338.

42 J. Steinkühler, P. De Tillieux, R. L. Knorr, R. Lipowsky and R. Dimova, Scientific Reports, 2018, 8, 11838.

43 Y. Okumura and Y. Iwata, Membranes, 2011, 1, 109-118.

44 Y. Okumura and S. Oana, Membranes, 2011, 1, 345-353.

45 V. Pereno, D. Carugo, L. Bau, E. Sezgin, J. Bernardino De La Serna, C. Eggeling and E. Stride, ACS Omega, 2017, 994-1002.

46 J. A. Bellon, M. J. Pino and N. Wilke, HardwareX, 2018, 4, e00037.

47 H. G. Behuria, B. K. Biswal and S. K. Sahu, Journal of Liposome Research, 2020, 255-266.

48 Y. Li, J. Jing, X. Zhang, J. Cao, Y. Li and S. Zhan, Electrochemistry Communications, 2012, 25, 151-154.

49 S. Tharad, C. Tangsongcharoen, P. Boonserm, J. L. Toca-Herrera and K. Srisucharitpanit, AIMS Biophysics, 2020, 7, $133-143$.

50 T. P. Dao, M. Fauquignon, F. Fernandes, E. Ibarboure, A. Vax, M. Prieto and J. F. Le Meins, Colloids and Surfaces A: Physicochemical and Engineering Aspects, 2017, 533, 347-353.

51 E. Rideau, F. R. Wurm and K. Landfester, Advanced Biosystems, 2019, 3, 1800324.

52 C. Herold, G. Chwastek, P. Schwille and E. P. Petrov, Langmuir, 2012, 28, 5518-5521.

53 C. V. Chrysikopoulos and P. Kruger, PhD thesis, Stanford University, 1986.

54 M. A. Malyarick, A. B. Ilyuhin and S. P. Petrosyants, Main Group Metal Chemistry, 1994, 17, 707-718.

55 T. Biver, R. Friani, C. Gattai, F. Secco, M. R. Tine and M. Venturini, The Journal of Physical Chemistry B, 2008, 112, $12168-12173$. 


\section{Electronic Supplementary Information (ESI)}

\section{Binding of His-tagged fluorophores to lipid bilayers and giant vesicles}

Shreya Pramanik ${ }^{a}$, Jan Steinkühler ${ }^{a, b}$, Rumiana Dimova ${ }^{a}$, Joachim Spatz $^{c}$, and Reinhard Lipowsky ${ }^{a, *}$

${ }^{a}$ Theory and Bio-Systems, Max Planck Institute of Colloids and Interfaces, Science Park Golm, 14424 Potsdam, Germany

${ }^{b}$ Center for Synthetic Biology, Northwestern University, Evanston, USA

${ }^{c}$ Max Planck Institute for Medical Research, 69120 Heidelberg, Germany

This Electronic Supplementary Information contains the Supplementary Figure S1 as well as the Tables $\mathbf{S 1}-\mathbf{S 5}$.
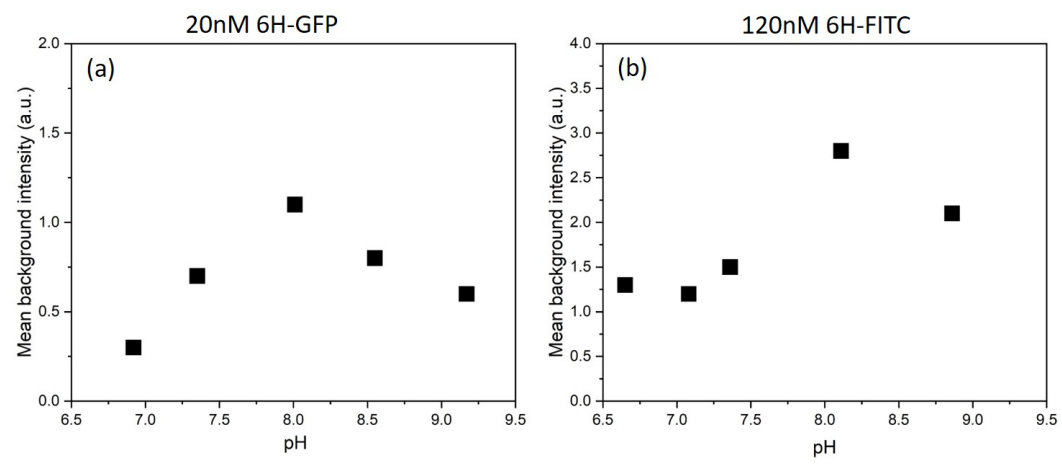

Figure S1: Dependence of background fluorescence intensity on the $\mathrm{pH}$ value in the absence of lipid vesicles: Mean background intensity for a solution of (a) $20 \mathrm{nM} 6 \mathrm{H}-$ GFP and of (b) $120 \mathrm{nM} 6 \mathrm{H}-\mathrm{FITC}$. In both cases, the background intensity has been averaged over the same partial field of view. 
Table S1: Statistics for the surface coverage of GUV membranes by $6 \mathrm{H}-\mathrm{GFP}$ as obtained for three different preparation methods, corresponding to the data in Figure 5. The membranes contained $3 \mathrm{~mol} \%$ NTA lipids and were exposed to a $20 \mathrm{nM}$ solution of $6 \mathrm{H}$ GFP at $\mathrm{pH}$ 7.45. The numerical values for the mean value and the standard deviation (SD) of the coverage are given in units of membrane-bound molecules per $\mu \mathrm{m}^{2}$.

\begin{tabular}{lcr}
\hline Preparation method & Mean coverage & SD \\
\hline Pt wire electroformation & 1084.4 & 573.9 \\
PVA hydrogel swelling & 781.7 & 471.4 \\
ITO glass electroformation & 27.5 & 12.5 \\
\hline
\end{tabular}

Table S2: Statistics for the surface coverage of GUV membranes by $6 \mathrm{H}-\mathrm{FITC}$ for three different preparation methods, corresponding to the data in Figure 6. The membranes contained $3 \mathrm{~mol} \%$ NTA lipids and were exposed to a $120 \mathrm{nM}$ solution of $6 \mathrm{H}-\mathrm{FITC}$ at $\mathrm{pH}$ 7.45. The numerical values for the mean value and the standard deviation (SD) of the coverage are given in units of membrane-bound molecules per $\mu \mathrm{m}^{2}$.

\begin{tabular}{lcr}
\hline Preparation method & Mean Coverage & SD \\
\hline Pt wire electroformation & 1409.9 & 624 \\
PVA hydrogel swelling & 416.3 & 144.4 \\
ITO glass electroformation & 18 & 15.5 \\
\hline
\end{tabular}

Table S3: Statistics for the surface coverage of GUV membranes by $6 \mathrm{H}-\mathrm{GFP}$ for two different electroformation methods, corresponding to the data in Figure 7. The membranes were prepared with $10 \mathrm{~mol} \%$ or $30 \mathrm{~mol} \%$ NTA lipids and exposed to a $20 \mathrm{nM}$ solution of $6 \mathrm{H}-\mathrm{GFP}$. The numerical values for the mean value and the standard deviation of the coverage are given in units of membrane-bound molecules per $\mu \mathrm{m}^{2}$.

\begin{tabular}{lrr}
\hline & $10 \%$ DGS-NTA(Ni) & 30\% DGS-NTA(Ni) \\
\hline Pt wire electroformation & $23050.28 \pm 6331.0$ & $23909.8 \pm 3366.0$ \\
ITO glass electroformationn & $1151.4 \pm 2455.9$ & $6455.0 \pm 3263.9$ \\
\hline
\end{tabular}


Table S4: Statistics for the surface coverage of GUV membranes by $6 \mathrm{H}-\mathrm{GFP}$ for different $\mathrm{pH}$ values in the exterior solution, corresponding to the data in Figure 9a. The membranes were prepared by platinum wire electroformation with 3 mol\% NTA lipids and were exposed to a $20 \mathrm{nM}$ solution of $6 \mathrm{H}-\mathrm{GFP}$. The $\mathrm{pH}$ was varied by the addition of $\mathrm{NaOH}$ solution. The numerical values for the mean value and the standard deviation (SD) of the coverage are given in units of membrane-bound molecules per $\mu \mathrm{m}^{2}$.

\begin{tabular}{lcr}
\hline $\mathrm{pH}$ & Mean Coverage & SD \\
\hline 6.71 & 6198.8 & 1009.3 \\
7.08 & 5982.8 & 1000.8 \\
7.15 & 6204.9 & 1452.3 \\
7.28 & 8202.7 & 1271,2 \\
7.42 & 5399.4 & 1345.4 \\
7.72 & 5536.6 & 2129.3 \\
8.25 & 2832.2 & 1249.9 \\
8.52 & 2935.6 & 482.2 \\
8.95 & 4045.9 & 616.5 \\
\hline
\end{tabular}

Table S5: Statistics for the surface coverage of GUV membranes by 6 H-FITC for different $\mathrm{pH}$ values in the exterior solution, corresponding to the data in Figure $9 \mathrm{~b}$. The membranes were prepared by platinum wire electroformation with $3 \mathrm{~mol} \%$ NTA lipids and were exposed to a $120 \mathrm{nM}$ solution of $6 \mathrm{H}$-FITC. The $\mathrm{pH}$ was varied by the addition of $\mathrm{NaOH}$ solution. The numerical values for the mean value and the standard deviation (SD) of the coverage are given in units of membrane-bound molecules per $\mu \mathrm{m}^{2}$.

\begin{tabular}{lcr}
\hline $\mathrm{pH}$ & Mean Coverage & SD \\
\hline 6.71 & 228.9 & 114.0 \\
7.08 & 109.1 & 29.6 \\
7.17 & 243.8 & 103.9 \\
7.38 & 244.8 & 50.6 \\
7.44 & 284.1 & 81.9 \\
7.53 & 310.9 & 88.4 \\
7.59 & 683.0 & 136.0 \\
7.77 & 911.4 & 106.4 \\
7.87 & 2403.4 & 718.1 \\
7.99 & 5145.7 & 620.3 \\
8.38 & 8727.4 & 1659.6 \\
8.52 & 8027.0 & 2465.0 \\
9.16 & 3605.0 & 1115.5 \\
\hline
\end{tabular}

